ARTICLE

\title{
Directed evolution of prenylated FMN-dependent Fdc supports efficient in vivo isobutene production
}

Annica Saaret (10 1, Benoît Villiers ${ }^{2 凶}$, François Stricher (10 2, Macha Anissimova (1) ${ }^{2}$, Mélodie Cadillon², Reynard Spiess ${ }^{1}$, Sam Hay (i) ${ }^{1} \&$ David Leys ${ }^{1 \times}{ }^{1 凶}$

Isobutene is a high value gaseous alkene used as fuel additive and a chemical building block. As an alternative to fossil fuel derived isobutene, we here develop a modified mevalonate pathway for the production of isobutene from glucose in vivo. The final step in the pathway consists of the decarboxylation of 3-methylcrotonic acid, catalysed by an evolved ferulic acid decarboxylase (Fdc) enzyme. Fdc belongs to the prFMN-dependent UbiD enzyme family that catalyses reversible decarboxylation of (hetero)aromatic acids or acrylic acids with extended conjugation. Following a screen of an Fdc library for inherent 3-methylcrotonic acid decarboxylase activity, directed evolution yields variants with up to an 80-fold increase in activity. Crystal structures of the evolved variants reveal that changes in the substrate binding pocket are responsible for increased selectivity. Solution and computational studies suggest that isobutene cycloelimination is rate limiting and strictly dependent on presence of the 3-methyl group.

\footnotetext{
${ }^{1}$ Department of Chemistry, Manchester Institute for Biotechnology, University of Manchester, Manchester, UK. ${ }^{2}$ Global Bioenergies, Evry-Courcouronnes, France.

凶email: benoit.villiers@global-bioenergies.com; david.leys@manchester.ac.uk
} 
T he irrefutable harmful environmental effects and depleting reserves of fossil fuels have powered an extensive amount of research to seek sustainable alternatives for the production of petrochemicals, including the gaseous alkene isobutene ${ }^{1-3}$. Due to the favourable reactivity, isobutene is widely used as a building block for fuel additives, rubber, plastic and a broad range of fine chemicals. Over 10 million tons of isobutene are produced every year, primarily by steam cracking crude oil. Low levels of microbial production of isobutene were first detected in the $1980 \mathrm{~s}^{4-6}$. More recently, isobutene production via a modified mevalonate (MVA) pathway using mevalonate diphosphate decarboxylase (MVD) to decarboxylate 3-hydroxyisovaleric acid was reported ${ }^{7}$ and subsequently patented ${ }^{8}$ (Fig. 1A). Further studies highlighted a more efficient route using mevalonate-3-kinase (M3K, Picrophilus torridus) that catalyses isobutene formation through an unstable phosphorylated intermediate ${ }^{9,10}$. The highest reported whole-cell isobutene production rate of $507 \mathrm{pmol} \mathrm{min}^{-1} \mathrm{~g} \mathrm{cells}^{-1}$ was reached using $E$. coli engineered with $\mathrm{M} 3 \mathrm{~K}$, however, this remains about $10^{5}$-fold lower than is economically viable ${ }^{7,9}$. The slow conversion could be surpassed by an alternative route, such as the more direct conversion of methylcrotonyl-CoA to isobutene through a combination of a thioesterase with a non-oxidative decarboxylase. The prenylated flavin (prFMN)-dependent ferulic acid decarboxylases (Fdc) catalyse reversible non-oxidative (de) carboxylation of a range of acrylic acids with extended conjugation $^{11-14}$. Recently, a reversible 1,3-dipolar cycloaddition mechanism was conclusively shown to underpin catalysis in Aspergillus niger $\mathrm{Fdc}(A n \mathrm{Fdc})^{11,15-17}$. First, the cycloaddition of the substrate results in cycloadduct Int1 (Fig. 1B). Decarboxylation occurs concomitantly with ring opening to form Int2. Following the exchange of $\mathrm{CO}_{2}$ with E282, protonation by E282 results in cycloadduct Int3 that releases the product through cycloelimination. Cycloadduct strain, mediated by a clash between the substrate $\mathrm{R}$ group and enzyme residues is key in ensuring reversible 1,3-dipolar cycloaddition ${ }^{17}$. Recent studies have shown rational engineering of $A n \mathrm{Fdc}$ can expand substrate scope to include aromatic substrates such as naphthoic acid ${ }^{18}$. Crotonic acid was found to inhibit $A n F d c$ by apparently irreversibly binding to the prFMN cofactor ${ }^{17}$. However, acrylic acid substrates lacking extended conjugation have rarely been reported in the wider UbiD enzyme family. Arguably, the natural UbiD substrate closest to 3-methylcrotonic acid is trans-anhydromevalonate 5-phosphate (tAHMP), which is decarboxylated by a UbiD-decarboxylase from a hyperthermophilic archaeon Aeropyrum pernix in an alternative mevalonate pathway ${ }^{19}$. Both 3-methylcrotonic acid and tAHMP contain a secondary beta carbon and lack extended conjugation, however, the phosphate group in tAHMP may facilitate strain manipulation in cycloadduct intermediates. A recent communication describes a pathway for the production of butadiene in E. coli where the decarboxylation of cis,cis-muconic acid is catalysed by Fdc from Saccharomyces cerevisiae enhanced by rational design ${ }^{20}$.

Herein, we report on discovery and optimization through directed evolution of Fdc decarboxylation activity with 3-methylcrotonic acid to produce isobutene. We seek to understand how a substrate lacking extended conjugation and bulk can be decarboxylated by Fdc, especially in view of the fact crotonic acid acts as an inhibitor of prFMN. We discuss the structural basis for an increase in activity and selectivity in Trichoderma atroviride $\mathrm{Fdc}(\mathrm{TaFdc})$ evolved by directed evolution. Surprisingly, the optimized variants remain unable to decarboxylate crotonic acid, suggesting that in the case of the substrate 3-methylcrotonic acid the single additional methyl group plays a key role in the cycloelimination process. Computational studies are used to rationalize the effect of the 3-methyl substitution on product formation.

\section{Results and discussion}

Initial screening of Fdc homologues. Initial in vivo screening tested $15 \mathrm{UbiD}$ homologues co-expressed with UbiX (E. coli K12) in E. coli for conversion of 3-methylcrotonic acid into isobutene as detected by gas chromatography. TaFdc exhibited over twice the isobutene production compared to other homologues
A

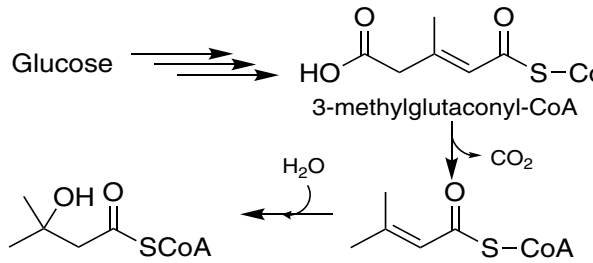

3-hydroxy-isovaleryl-CoA

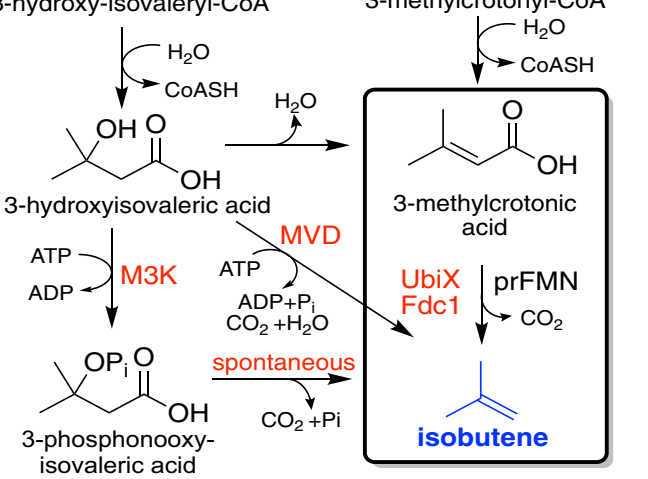

B

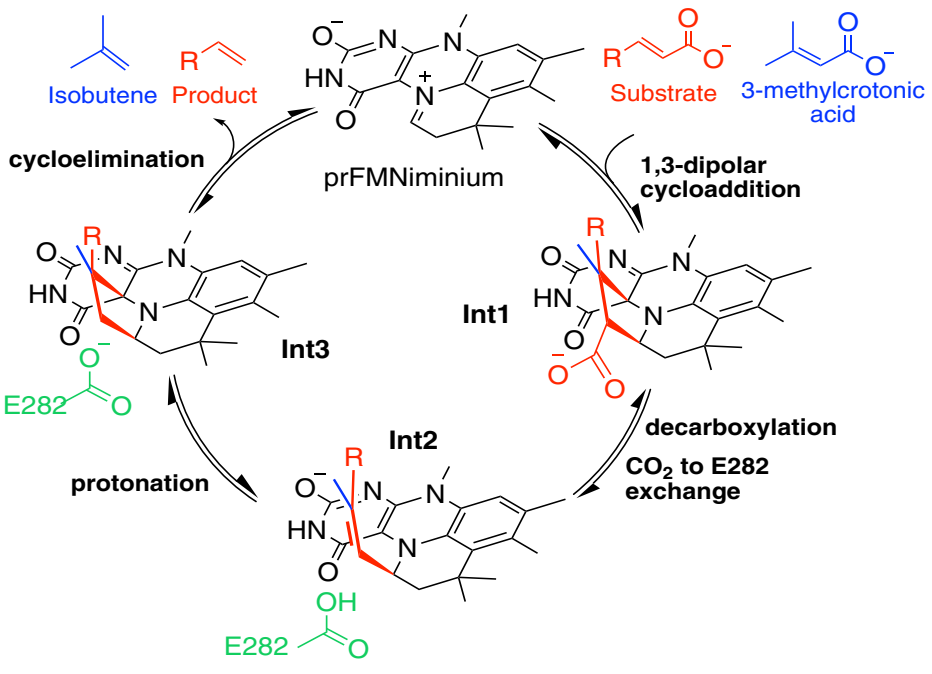

Fig. 1 Isobutene production via the modified mevalonate pathway utilizing Fdc for the last step. A Routes to isobutene via modified mevalonate pathways. Previously published mevalonate diphosphate decarboxylase (MVD) ${ }^{7}$ and mevalonate-3-kinase (M3K) ${ }^{9}$ produce isobutene via 3-hydroxyisovaleric acid. Fdc1, co-expressed with UbiX, catalyses the decarboxylation of 3-methylcrotonic acid to give isobutene. B Fdc decarboxylation reaction mechanism with 3-methylcrotonic acid in blue and common Fdc substrates with a conjugated R-group in red. First, the 1,3-dipolar cycloaddition of the substrate to prFMNiminium leads to the first pyrrolidine cycloadduct, Int1. Decarboxylation and ring-opening forms the noncyclic alkene adduct Int2. Protonation by a conserved glutamic acid residue yields the second pyrrolidine cycloadduct Int3 followed by cycloelimination to give the product. 
Table 1 Summary table of AnFdc and TaFdc variants.

\begin{tabular}{|c|c|c|c|c|c|c|c|}
\hline & AnFdc & AnFdcl & AnFdcll & TaFdc & TaFdcl ${ }^{\mathrm{a}}$ & TaFdcVa & TaFdcII \\
\hline Mutations & WT & T395M & $\begin{array}{l}\text { T395M } \\
\text { R435P P438W }\end{array}$ & WT & $\mathrm{T} 405 \mathrm{M}$ & $\begin{array}{l}\text { E25N N31G G305A D351R K377H P402V } \\
\text { F404Y T405M T429A V445P Q448W }\end{array}$ & $\begin{array}{l}\text { F404Y T405M } \\
\text { V445P Q448W }\end{array}$ \\
\hline $\begin{array}{l}\text { Crystal } \\
\text { structure }\end{array}$ & 4ZA4 & 7NF3 & $7 N F 4$ & 7NEY & $\begin{array}{l}\text { No crystal } \\
\text { structure }\end{array}$ & 7NF1 & No crystal structure \\
\hline
\end{tabular}

(Supplementary Table 1) and a directed evolution approach was taken to generate a variant of $\mathrm{TaFdc}$ with superior isobutene production activity and selectivity for 3-methylcrotonic acid over cinnamic acid (schematically presented in Supplementary Fig. 1). TaFdcI, with a T405M mutation, was the first variant with a considerable increase in isobutene. $\mathrm{TaFdcV}$ generated by 4 rounds of evolution has 11 mutations: E25N, N31G, G305A, D351R, K377H, P402V, F404Y, T405M, T429A, V445P and Q448W (Table 1).

Characterization of $\mathbf{T a F d c}$ and $\mathrm{TaFdcV}$. TaFdc wild-type and $\mathrm{TaFdcV}$ with an N-terminal hexa-histidine tag were co-expressed with E. coli K-12 UbiX in E. coli and purified with Ni-NTA resin. UV-Vis spectra of both purified proteins exhibit a distinct peak at $380 \mathrm{~nm}$, thought to correspond to the cofactor active form prFMNiminium (Supplementary Fig. 2A) ${ }^{16}$. ESI-MS confirmed the presence of prFMNiminium in both enzyme variants (Supplementary Fig. 2B and C). The shape of the $380 \mathrm{~nm}$ peak and cofactor content (assessed by the ratio of absorbances at 280 and $380 \mathrm{~nm}$ ) varied from batch to batch.

TaFdc showed decarboxylation activity with cinnamic and sorbic acid, with rates $k_{\mathrm{obs}}=7.2 \pm 0.3$ and $3.2 \pm 0.3 \mathrm{~s}^{-1}$, respectively (reported for a batch with a $380: 280 \mathrm{~nm}$ ratio of 0.067 ). These values are comparable to those reported for $A n \mathrm{Fdc}^{11,16}$. In contrast, the $\mathrm{TaFdcV}$ variant showed compromised activity with sorbic acid $\left(k_{\mathrm{obs}}=0.33 \pm 0.03 \mathrm{~s}^{-1}\right)$ and no activity was detected with cinnamic acid. When exposed to light, TaFdc sorbic acid decarboxylation activity steadily deteriorates with a half-life of $1 \mathrm{~h}$ compared to enzyme stored in dark (Supplementary Fig. 2H). This is consistent with Fdc light-sensitivity as described previously ${ }^{17}$. Upon irradiation with a $405 \mathrm{~nm}$ LED lamp, the characteristic $380 \mathrm{~nm}$ peak in the UV-visible absorbance spectra of $\mathrm{TaFdc}$ and $\mathrm{TaFdcV}$ irreversibly splits to peaks at 365 and $425 \mathrm{~nm}$ (Supplementary Fig. 2I and J).

Incubation of both $\mathrm{TaFdc}$ and $\mathrm{TaFdcV}$ with 3-methylcrotonic acid triggered a change in the protein UV-Vis spectrum to reveal peaks at 340 and $425 \mathrm{~nm}$, suggestive of a covalent substrate:prFMN adduct accumulating under turnover conditions. Following a desalting step, the spectrum returns to the as-isolated $380 \mathrm{~nm}$ single feature, confirming that a long-lived, inhibitory covalent complex with 3-methylcrotonic acid is not formed (Supplementary Fig. 2D and E). Incubation of $80 \mu \mathrm{mol} \mathrm{TaFdcV}$ with $10 \mathrm{mM}$ 3-methylcrotonic acid led to a complete shift in the corresponding $\mathrm{UV}-\mathrm{V}$ is spectrum. In contrast, the wild-type $\mathrm{TaFdc}$ required prolonged incubation with $50 \mathrm{mM}$ 3-methylcrotonic acid to achieve full spectral conversion, suggesting a substantially higher $K_{\mathrm{D}}$ and/or adduct formation rate for the wild-type enzyme. An ESI-MS spectrum of the desalted sample showed peaks corresponding to both prFMNiminium and a putative Int3 prFMN cycloadduct with 3-methylcrotonic acid (Supplementary Fig. 3). This may be due to a small proportion of 3-methylcrotonic acid remains bound to prFMN as Int3, suggesting Int3 elimination is the rate-limiting step, or that a proportion of the Int3 species has irreversibly isomerized to a more stable conformation.

In order to assess the scope for activity with acrylic acids lacking extended conjugation, $\mathrm{TaFdc}$ and $\mathrm{TaFdcV}$ were incubated with trans-2-pentenoic and trans-2-hexenoic acid, compounds that have previously been reported to undergo some AnFdcmediated decarboxylation ${ }^{11}$. UV-Vis absorbance spectra indicated that TaFdc bound both acids (Supplementary Fig. 2F), whereas the $T a F d c V$ variant preferred the smaller pentenoic acid and required higher concentrations to fully bind hexenoic acid (Supplementary Fig. 2G). In contrast to samples incubated with 3-methylcrotonic acid, the UV-Vis spectra of samples incubated with pentenoic or hexenoic acid were unaffected by a desalting step, indicating that pentenoic and hexenoic acid irreversibly binds to $T a F d c / T a F d c V$. Quantitative GC assay indicates that pentene production from hexenoic acid by $A n F d c$ is limited to a single turnover (Supplementary Fig. 4).

Crystal structures of $T a F d c$ and $T a F d c V$ reveal mutation impact on the substrate-binding pocket. In order to understand how $\mathrm{TaFdcV}$ mutations aid in isobutene production, crystal structures of $\mathrm{TaFdc}$ and $\mathrm{TaFdcV}$ were solved at a resolution of 1.74 and $1.89 \AA$, respectively. An overlay of the wild-type and the variant crystal structures shows that the key residues F447, Q200 and the catalytic network of E287-R183-E292 are unaffected by the mutations (Fig. 2A) ${ }^{16}$. The T405M mutation is located at the active site, extending towards the space above the prFMN uracil ring while the Q448W and F404Y mutations are situated in the second shell from the active site. The E292 residue side chain occupies 'up' and 'down' conformations, while weak electron density suggests a high degree of mobility for the L449 side chain. The mobile E292 and L449 gate access to the active site (Fig. 2B) while the $\mathrm{Q} 448 \mathrm{~W}$ mutation in $\mathrm{TaFdcV}$ narrows the binding pocket (Fig. 2C). The T405M and Q448W mutations are likely to be responsible for the increased selectivity for 3-methylcrotonic acid in $\mathrm{TaFdcV}$ by enhancing the substrate/active site shape complementarity, blocking access to larger substrates (Supplementary Fig. 5). While comparison of $\mathrm{TaFdc}$ and $\mathrm{TaFdcV}$ crystal structures reveals the basis for increased selectivity in the evolved enzyme, it is not immediately clear why 3-methylcrotonic acid can yield isobutene from Int3.

Formation of stable cycloadducts with inhibitors. The effects of crotonic and 2-butynoic acid on $\mathrm{TaFdcV}$ were studied to determine whether the mutations that increase in 3-methylcrotonic acid turnover also affected activity with related compounds. Incubation of $\mathrm{TaFdcV}$ with 2-butynoic and crotonic acid led to the familiar split of the $380 \mathrm{~nm}$ prFMN peak in the UV-Vis spectrum (Fig. 3A and D), similar to 3-methylcrotonic acid. However, as previously observed with pentenoic and hexenoic acid, the spectrum did not recover the following desalting, suggesting that a covalent inhibitory adduct is formed. Similar trends were observed with $\mathrm{TaFdc}$, however, incubation at higher inhibitor concentration was required to drive changes in the UV-Vis spectrum.

Upon addition of crotonic acid, a gradual shift in UV-Vis spectrum occurs over minutes, allowing estimation of adduct formation rate (Supplementary Fig. 6A). The observed rate remains first order with respect to crotonic acid, with $k_{\text {obs }}=0.34$ $\pm 0.03 \mathrm{~min}^{-1}$ at the highest concentration tested $(50 \mathrm{mM})$ 

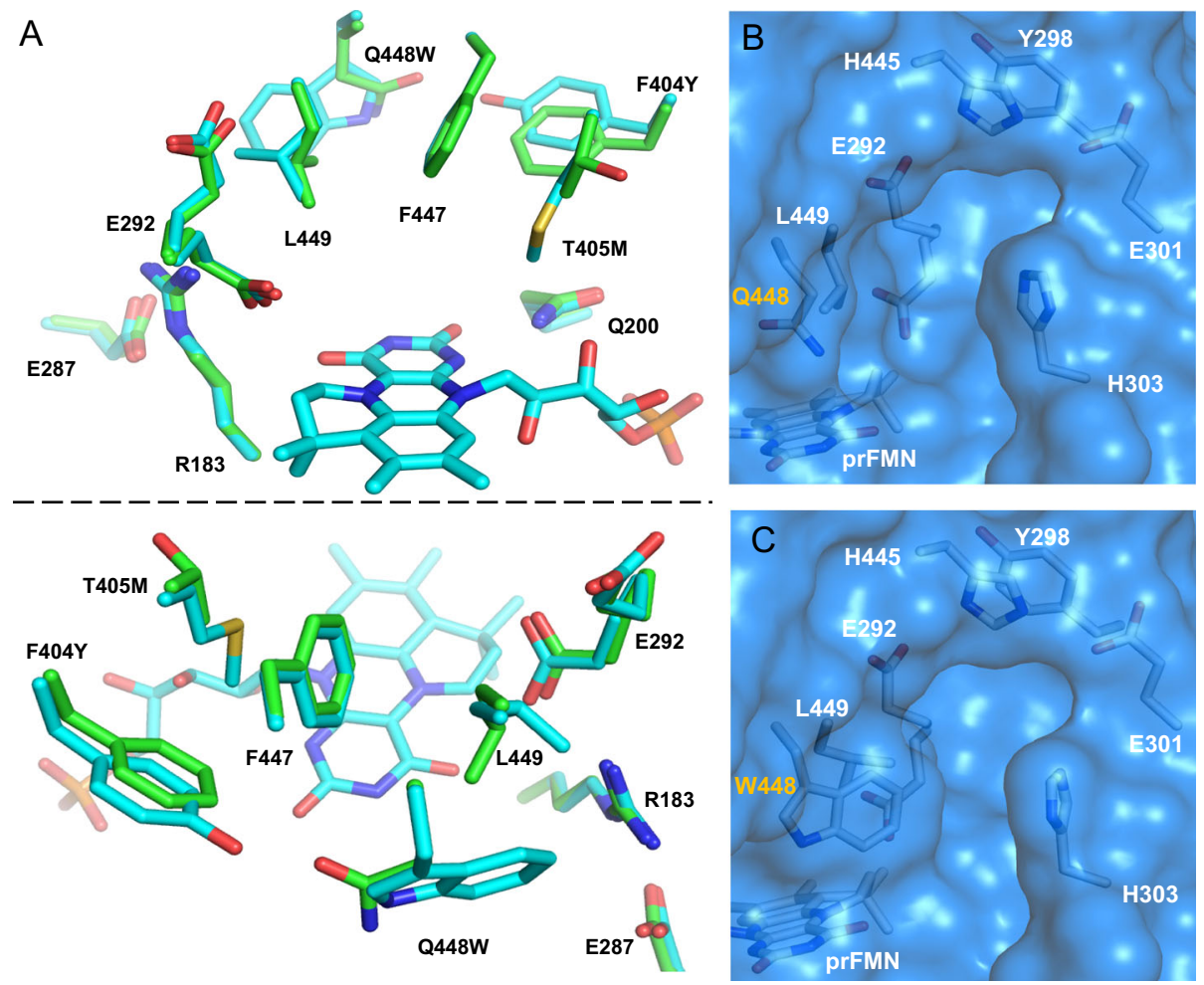

Fig. 2 Comparison of TaFdc and TaFdcV. A Two views of the overlay of TaFdc wild-type (green, 7NEY [10.2210/pdb7NEY/pdb]) and TaFdcV (blue, 7NFO $[10.2210 / \mathrm{pdb} 7 \mathrm{NFO} / \mathrm{pdb}]$ ) active sites. Comparison of TaFdc B and TaFdcV C binding pockets. The mobile L449 and E292 gate the entrance to the active site and the Q448W mutation narrows the entrance to the active site.

(Supplementary Fig. 6B). In contrast, a similar shift in UV-Vis spectrum upon addition of the substrate 3-methylcrotonic acid occurs rapidly within seconds, and at substantially lower 3-methylcrotonic acid concentrations. This suggests that crotonic acid adduct formation is hindered by a higher $K_{\mathrm{D}}$ and/or slower rate of cycloaddition.

ESI-MS and co-crystallization studies confirmed that the $\mathrm{TaFdcV}$ 2-butynoic acid adduct stalls as Int1, while the $\mathrm{TaFdcV}$ crotonic acid adduct undergoes decarboxylation to stall at the Int3 species (Fig. 3). Similar behaviour has been reported for $A n \mathrm{Fdc}^{17}$. No decarboxylation of the Int1 with 2-butynoic acid was detected, even in 1-month-old crystals. In contrast, although only Int3 was observed in co-crystals with crotonic acid, ESI-MS also showed a peak for the corresponding Int1 (Fig. 3E). It is unclear whether Intl can be detected in this case because decarboxylation of crotonic acid is slow, or because there is an equilibrium between Int1 and Int3 at ambient $\mathrm{CO}_{2}$ levels.

$A n$ FdcII with three point-mutations has an identical active site conformation to $\mathbf{T a F d c V}$. To further understand how the architecture of the active site affects the decarboxylation of 3-methylcrotonic acid, corresponding key mutations from $\mathrm{TaFdcV}$ were introduced in $A n \mathrm{Fdc}$. $A n \mathrm{Fdc}$ has been established as a model system due to the fact that it readily yields atomic resolution crystal structures ${ }^{11,16-18}$. Two variants were studied: AnFdc T395M (AnFdcI) and the triple mutant AnFdc T395M R435P P438W (AnFdcII). Overlay of the AnFdc wild-type and TaFdcV crystal structures reveals a downward shift of the Y404 residue in $\mathrm{TaFdcV}$ in the secondary shell compared to the corresponding Y394 in AnFdc (Fig. 4A). The Y394 residue is unaffected in the $A n$ FdcI variant compared to wild-type (Fig. 4B). In contrast, the active site of the $A n \mathrm{FdcII}$ variant matches that of $\mathrm{TaFdcV}$ in the conformation of Y394 and M395 (Fig. 4C).
As expected, neither $A n$ FdcI nor $A n$ FdcII were active with cinnamic acid, likely due to a clash between the substrate phenyl ring and M395. While binding of crotonic acid in $A n F d c$ wildtype cannot be detected by the UV-Vis spectra over 2-h incubation, both mutants $A n \mathrm{FdcI}$ and $A n \mathrm{FdcII}$ readily bind the inhibitor, evident from UV-Vis spectra, demonstrating increased selectivity towards smaller substrates.

TaFdcII has comparable isobutene production activity to TaFdcV. Selected $T a F d c$ variants (wild-type, $T a F d c I$ i.e. T405M, TaFdcV, TaFdcII, see Supplementary Fig. 1) and AnFdc variants (wild-type, $A n \mathrm{FdcI}, A n \mathrm{FdcII}$ ) were purified and assayed for isobutene production. TaFdcII (F404Y, T405M, V445P, Q448W) was created by rational design based on the structural analysis of TaFdc wild-type, $T a F d c V$ and $A n F d c I I$. A comparison of the isobutene titre obtained following 2 and $4 \mathrm{~h}$ incubation revealed TaFdcI and $A n F d c I$ produced 4-9 times the amount of isobutene compared to the wild-type enzymes. Additional mutations in $A n$ FdcII and TaFdcII led to a substantial further increase of 18 and 81 fold, respectively, in isobutene production (Supplementary Fig. 7). Surprisingly, the in vitro titer obtained with TaFdcII was slightly higher than the corresponding $\mathrm{TaFdcV}$ levels obtained (Fig. 5). Thus, the 4 point mutations in TaFdcII (F404Y, T405M, V445P, Q448W) and 3 point mutations in AnFdcII (T395M, R435P, P438W), that create an active site architecture identical to TaFdcV (Fig. 4), appear largely responsible for the increased isobutene activity compared to wild-type TaFdc and $A n F d c$.

While $A n F d c$ wild-type was included in the initial UbiD screen, the $A n \mathrm{Fdc}$ wild-type was 90 times lower in activity in vivo compared to TaFdc. Hence, $A n$ Fdc was not selected for further directed evolution, despite having comparable in vitro activity to TaFdc. The disparate and lower activity in vivo might be attributed to $A n F d c$-specific inhibition by metabolites such as phenylacetaldehyde ${ }^{11}$. An initial comparison of in vitro isobutene 
A

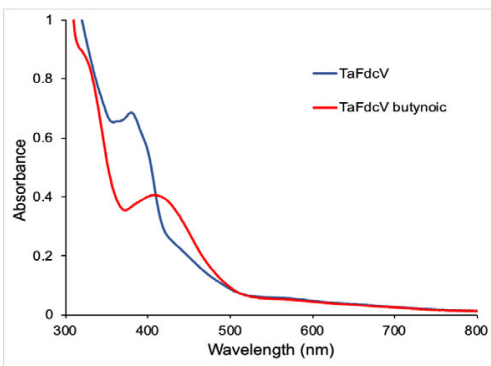

$\mathrm{D}$

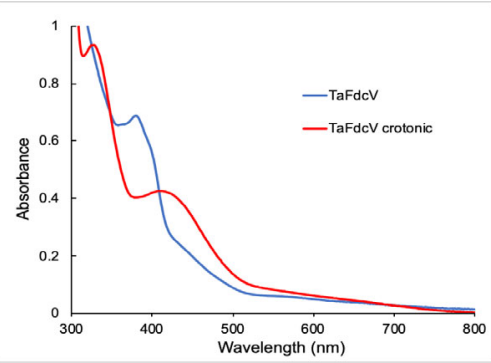

B

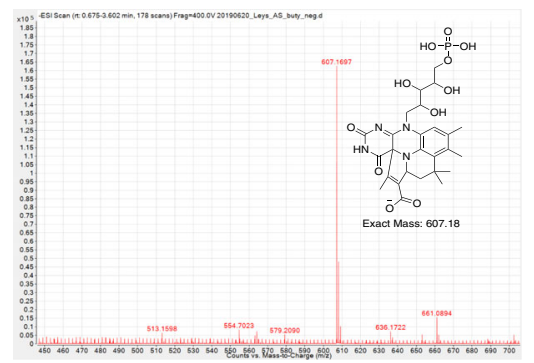

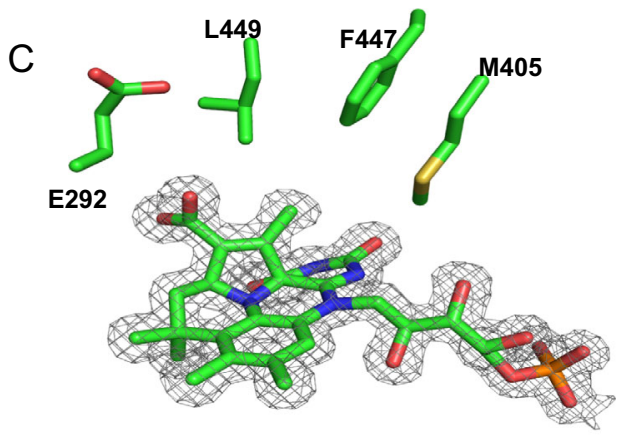

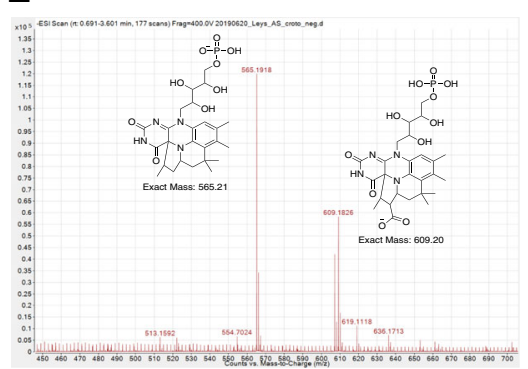

$\mathrm{F}$

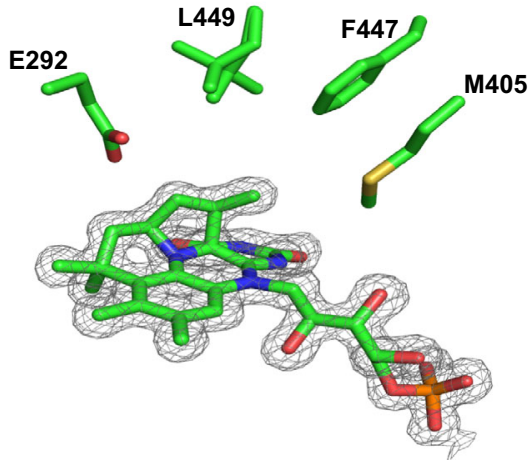

Fig. 3 Cycloadduct formation in TaFdcV with inhibitors butynoic and crotonic acid. A UV-Vis spectra of TaFdcV before and after incubating with 2-butynoic acid. B ESI-MS spectra of TaFdcV incubated with 2-butynoic acid and desalted, showing the formation of prFMN-butynoic adduct $[\mathrm{M}+\mathrm{H}]^{+}=607.18$. C Crystal structure of TaFdcV with prFMN-butynoic adduct (7NF1 [10.2210/pdb7NF1/pdb]). D UV-Vis spectra of TaFdcV as is and incubated with crotonic acid E ESI-MS spectra of $\mathrm{TaFdcV}$ incubated with crotonic acid (and desalted) showing the formation of decarboxylated prFMN-crotonic adduct $[\mathrm{M}-\mathrm{H}]^{-}=565.21$ and traces of prFMN-crotonic with the carboxylate group $[\mathrm{M}-\mathrm{H}]^{-}=609.20$. F Crystal structure of TaFdcV prFMN-crotonic adduct (7NF2 [10.2210/pdb7NF2/pdb]).

A

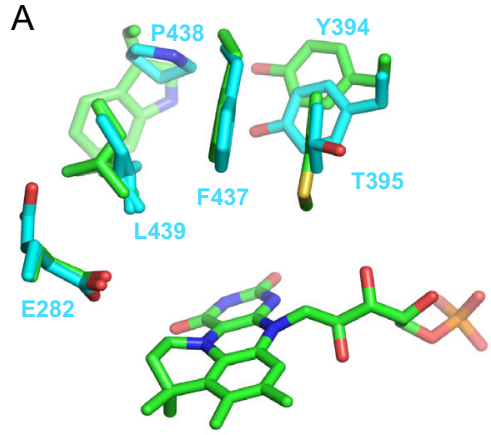

B

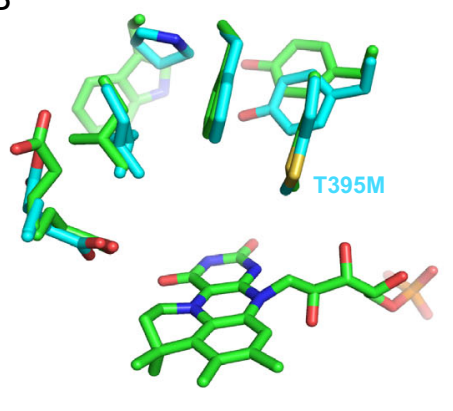

C

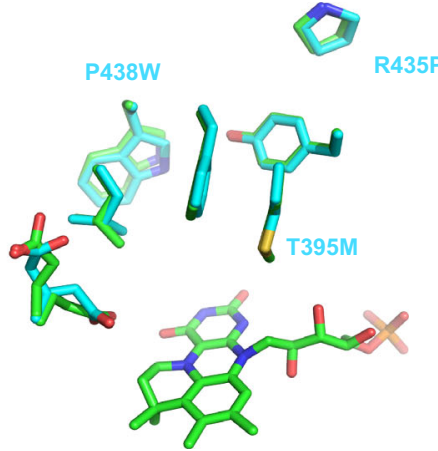

Fig. 4 Comparison of the TaFdcV and AnFdc wild-type and variant active sites. Overlay of TaFdcV (green, 7NEY [10.2210/pdb7NEY/pdb]) with A AnFdc wild-type (blue, 4ZA4 [10.2210/pdb4ZA4/pdb]), B AnFdcl (blue, 7NF3 [10.2210/pdb7NF3/pdb]) and C AnFdcll (blue, 7NF4 [10.2210/pdb7NF4/pdb]) crystal structures (AnFdc variant residue numbering according to AnFdc).

production levels using crude cell lysate from cells expressing $\mathrm{TaFdc}$ variants with those expressing MVD and/or M3K reveals a $\sim 50$-fold increase is observed for $\mathrm{TaFdcV}$ compared to MVD/ M3K levels (Supplementary Fig. 8). This demonstrates that the evolved $\mathrm{TaFdcV}$ is vastly superior in catalysing the decarboxylative step compared to the previously described enzyme systems.

Computational studies reveal a mechanistic basis for isobutene production. It is curious that a single methyl group difference, as occurs between crotonic acid and 3-methylcrotonic acid, determines whether the compound is a substrate or inhibitor for the evolved Fdc variants. The marked influence of the additional methyl group on Int3 cycloelimination suggests this step may proceed via a cationic or radical beta carbon stabilized through additional hyperconjugation effects. A density functional theory (DFT) active site 'cluster' model (Supplementary Fig. 9) was used to investigate why 3-methylcrotonic acid is decarboxylated and eliminated by $\mathrm{TaFdcV}$ in contrast to crotonic acid.

The potential energy surface for the cycloelimination of Int3 to the non-covalent product complex was computed for both crotonic acid and 3-methylcrotonic acid by varying the $\mathrm{C}_{\alpha}-\mathrm{C}_{1}$, and $\mathrm{C}_{\beta}-\mathrm{C}_{4 \mathrm{a}}$ bond lengths (Fig. 6). These suggest that 3-methylcrotonic acid undergoes a more asynchronous elimination, with the transition state $\mathrm{C}_{\alpha}-\mathrm{C}_{1^{\prime}}$ and $\mathrm{C}_{\beta}-\mathrm{C}_{4 a}$ bond lengths of 1.96 and $2.97 \AA$, respectively, compared to 1.95 and $2.77 \AA$ for crotonic acid, 


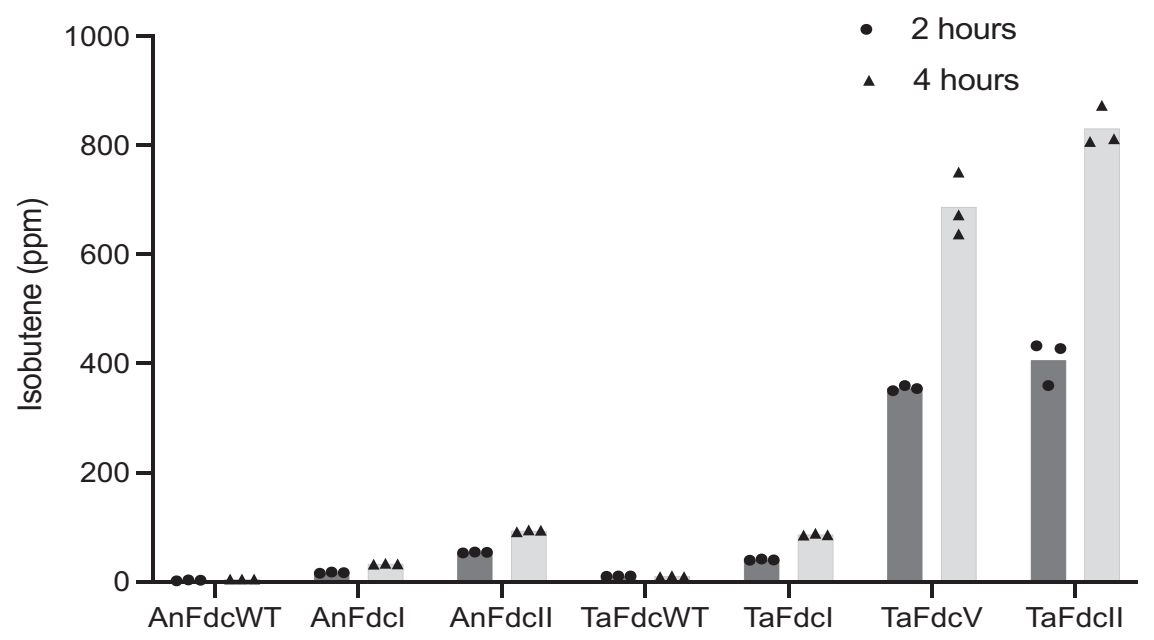

Fig. 5 Isobutene production by TaFdc and AnFdc variants. 3-methylcrotonate decarboxylation assay with purified enzyme comparing isobutene production as detected by GC by TaFdc and AnFdc variants (Table 1), both with N-terminal His-tags, over 2 and $4 \mathrm{~h}$ with $10 \mathrm{mM} 3-\mathrm{methylcrotonate}$ and $0.3 \mathrm{mg} / \mathrm{mL}$ enzyme. Fold increase comparison is shown in Supplementary Fig. 7. Source data are provided as a Source Data file.

respectively. This is linked to an increased charge separation occurring between the $\mathrm{C}_{\beta}$ and prFMN for the 3-methylcrotonic acid compared to crotonic acid (Supplementary Tables 4 and 5), possibly affected by additional hyperconjugation in the case of 3-methylcrotonic acid. The release of propene from crotonic acid Int3 cycloadduct is more endothermic by $\sim 8 \mathrm{~kJ} \mathrm{~mol}^{-1}$ and has a higher energy barrier by $19 \mathrm{~kJ} \mathrm{~mol}^{-1}$ compared to the release of isobutene from Int3 with 3-methylcrotonic acid. If the activation entropy is similar for the two reactions then the transition state energy difference translates to a 2200 slower rate for the release of propene from Int 3 at $293 \mathrm{~K}$, explaining the lack of crotonic acid turnover under conditions tested.

The limit of prFMN-dependent (de)carboxylation by UbiD enzymes. Directed evolution of $\mathrm{TaFdc}$ to $\mathrm{TaFdcV}$ resulted in a marked increase in activity with 3-methylcrotonic acid. Surprisingly, the evolved mutant remained unable to convert crotonic acid to the corresponding propene. This contrasts with previous evolved studies aimed at expanding the $A n F d c$ substrate repertoire to include (hetero)aromatic compounds ${ }^{18}$. In this case, the evolution of activity against heteroaromatic bicyclic compounds yielded a broad specificity variant able to convert even naphthoic acid. It is thus possible that 3-methylcrotonic acid represents a limit for bona fide UbiD-substrates, indicating that prFMNdependent catalysis requires more than an $\alpha, \beta$-unsaturated acrylic acid (i.e. a secondary $\mathrm{C}_{\beta}$ carbon) to yield reversible cycloelimination. Indeed, crotonic acid readily forms irreversible adducts with (evolved) Fdc that proceed to the last step prior to product formation. Detailed studies of the $A n F d c$ mechanism revealed considerable enzyme-induced strain in substrate-cofactor adducts that avoid dead-end local energy minima during the covalent catalysis ${ }^{17}$. In the case of smaller substrates such as (3-methyl) crotonic acid, the scope for enzyme-induced strain as a tool to optimize the energy landscape is minimal. In this case, cycloelimination of isobutene appears feasible at ambient conditions whereas propene production is not. Computational studies provide a rationale behind these observations, suggesting a $\sim 2200$ fold slower rate for the release of propene from Int3. Thus, further optimization of isobutene production and future evolution of propene producing Fdc variants will need to focus on the energetics of the hydrocarbon elimination step.

\section{Methods}

In vivo isobutene assay. In vivo screenings were carried out on a 96-well plate (DW96, $2.2 \mathrm{~mL}$ wells, sealed with a foil sheet). TaFdc and other UbiD homologues were co-expressed with UbiX (E. coli, K-12) in a petDuet vector (UbiD in MCS1 and UbiX in MCS2) in E. coli (BL21, DE3). Isobutene production from $0.4 \mathrm{~mL}$ reaction mix with $10 \mathrm{mM} 3$-methylcrotonic acid was detected from the headspace by gas chromatography. The GC method consisted of $100 \mu \mathrm{L}$ of headspace with a split ratio of 10 injected to RTX-1 column $(15 \mathrm{~m}, 0.32 \mathrm{~mm}$ internal diameter, $5 \mu \mathrm{m}$ film thickness, from RESTEK 10178-111) using nitrogen as a carrier gas $(1 \mathrm{~mL} / \mathrm{min}$ flow rate). The oven temperature was held at $100^{\circ} \mathrm{C}$ and the injector and detector were maintained at $250^{\circ} \mathrm{C}$. Isobutene was calibrated at 1000,5000 and $10,000 \mathrm{ppm}$ with standards from Messer.

Mutagenesis. Point mutations (TaFdcI, TaFdcII, $A n$ FdcI, $A n$ FdcII, Supplementary Table 6) were generated with a Q5 mutagenesis kit from New England Biolabs. Primers were designed with NEBaseChanger (New England Biolabs). The presence of the point mutation was confirmed by sequencing (Eurofins).

Protein expression. A pETDuet-1 vector containing genes for T. atroviride Fdc (with an N-terminal 6-histidine affinity tag) and UbiX (E. coli, K-12) was transformed into BL21(DE3) competent cells following the manufacturer's protocol (Novagen). A colony was inoculated into Lysogeny Broth (supplemented with $100 \mu \mathrm{g} / \mathrm{mL}$ ampicillin) and incubated by shaking overnight at $37^{\circ} \mathrm{C} .5 \mathrm{~mL}$ of LB culture was inoculated into $1 \mathrm{~L}$ of Terrific Broth (TB, Formedium), supplemented with $100 \mu \mathrm{g} / \mathrm{mL}$ ampicillin. The culture was incubated by shaking at $37^{\circ} \mathrm{C}$ until the optical density of $0.6-0.8$. The cells were induced with $0.4 \mathrm{mM}$ isopropyl $\beta$-D-1thiogalactopyranoside (IPTG) and supplemented with $0.5 \mathrm{mM} \mathrm{MnCl}_{2}$. The cultures were incubated by shaking at $18^{\circ} \mathrm{C}$ for $24 \mathrm{~h}$. The cells were harvested by centrifugation $(10 \mathrm{~min}, 8939 \times g)$ and frozen.

Protein purification. Frozen cells were supplemented with EDTA-free protease inhibitor mixture (Roche Applied Science), lysozyme, DNAse, and RNAse (Sigma) and resuspended (50 mM HEPES, $300 \mathrm{mM} \mathrm{KCl,} \mathrm{pH} 6.8$ ). The cells were lysed by sonication on ice (Bandelin Sonoplus sonicator, TT13/F2 tip, 30\% power with $20 \mathrm{~s}$ on/40 s off for $15 \mathrm{~min})$ and centrifuged $(1 \mathrm{~h}, 174,000-185,500 \times g$, Beckman Optima LE-80k ultracentrifuge, Ti50.2 rotor). The cell-free extract was loaded on Ni-NTA resin, washed with 4 column volumes of $40 \mathrm{mM}$ imidazole buffer $(40 \mathrm{mM}$ imidazole, $50 \mathrm{mM}$ HEPES, $300 \mathrm{mM} \mathrm{KCl}, \mathrm{pH}$ 6.8) and eluted in $1 \mathrm{~mL}$ fractions with $250 \mathrm{mM}$ imidazole buffer $(250 \mathrm{mM}$ imidazole, $50 \mathrm{mM}$ HEPES, $300 \mathrm{mM} \mathrm{KCl}, \mathrm{pH}$ 6.8). Fractions containing protein were combined and desalted into $25 \mathrm{mM}$ HEPES, $150 \mathrm{mM} \mathrm{KCl}, \mathrm{pH}$ 6.8. Exposure to light was minimized by covering with foil and using black Eppendorf tubes.

Cycloadduct formation. $\mathrm{TaFdcV}(500 \mu \mathrm{L}, 0.45 \mathrm{mM}$ protein, $25 \mathrm{mM}$ HEPES, $150 \mathrm{mM} \mathrm{KCl}, \mathrm{pH} 6.8$ ) was incubated with crotonic or 2-butynoic acid. The formation of the prFMN-crotonic acid cycloadduct was followed by UV-Vis spectroscopy and additional acid were added until full conversion (complete loss of the $380 \mathrm{~nm}$ peak). The protein was desalted to $25 \mathrm{mM}$ HEPES, $150 \mathrm{mM} \mathrm{KCl}, \mathrm{pH} 6.8$ and plated for crystal trials. 
A

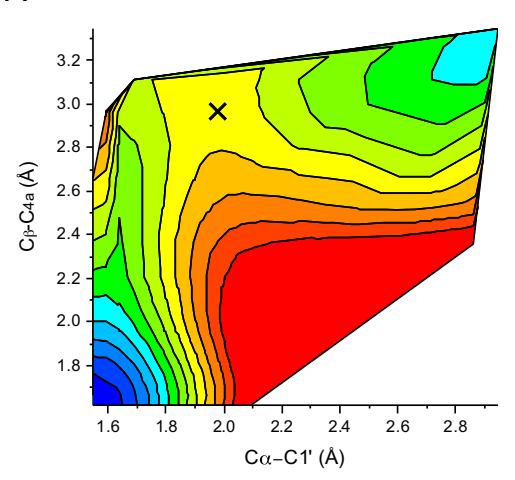

B

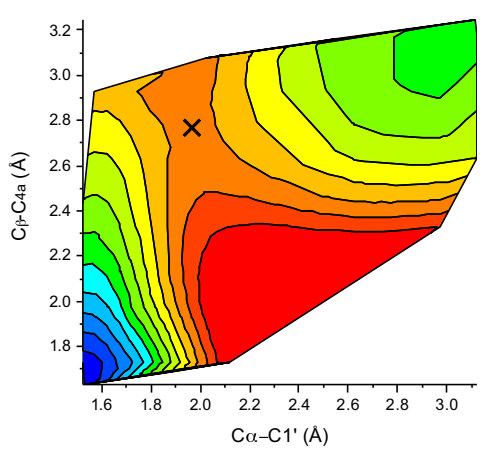

C
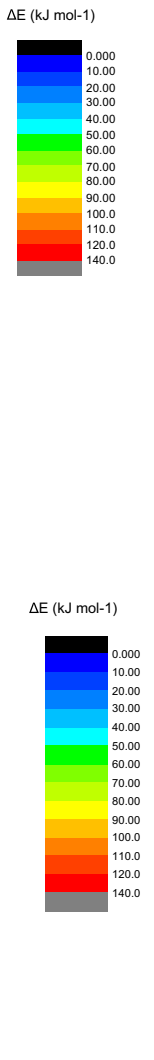

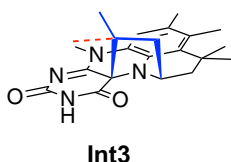

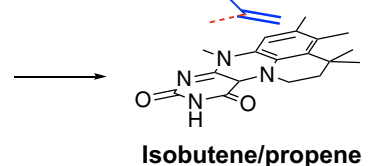

100

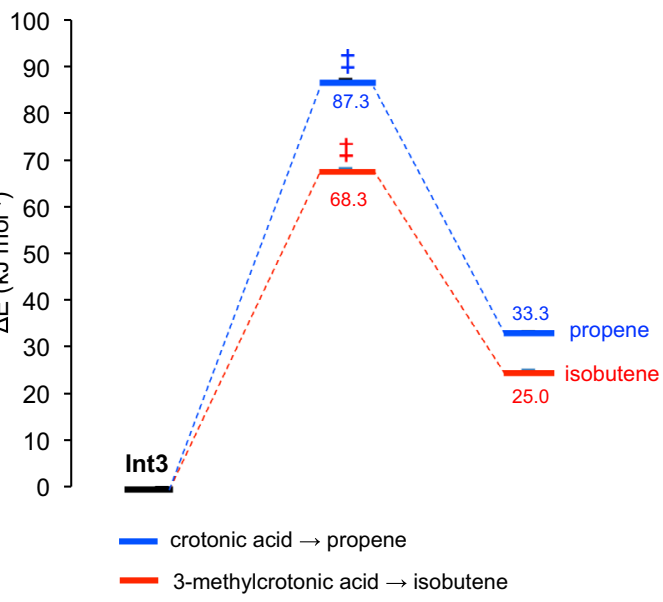

$\mathrm{D}$

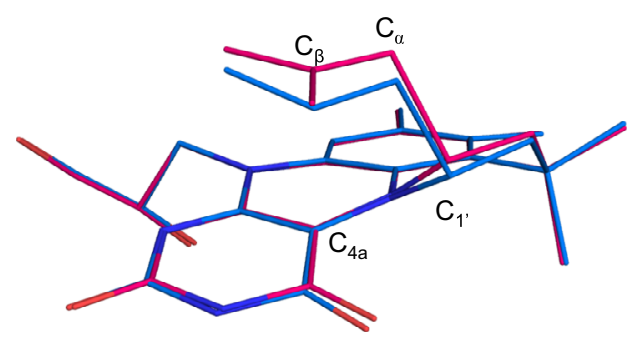

Fig. 6 DFT calculations applied to the active site of TaFdc with 3-methylcrotonic and crotonic acid. Contour map of the potential energy ( $\mathrm{kJ}$ mol-1) landscape for 3-methylcrotonic acid (A) and crotonic acid (B) conversion to isobutene and propene, respectively, from Int3 by TaFdcV, projected transition state is marked by X. C Zero-point energy corrected potential energy ( $\mathrm{kJ} \mathrm{mol}^{-1}$, Supplementary Tables 2 and 3) scheme for 3-methylcrotonic (red) and crotonic acid (blue) with the Int3 set as 0 and the projected approximate transition state denoted with double daggers. D overlay of the DFT optimized transition states between Int3 and product for 3-methylcrotonic (pink, $C_{\alpha}-C_{1}$ and $C_{\beta}-C_{4 a}$ bond lengths of 1.96 and $2.97 \AA$, respectively) and crotonic acid (blue, $\mathrm{C}_{\alpha}-\mathrm{C}_{1}$ and $\mathrm{C}_{\beta}-\mathrm{C}_{4 a}$ bond lengths of 1.95 and $2.77 \AA$, respectively). Source data underlying $\mathbf{A}-\mathbf{C}$ are provided as a Source Data file.

\section{Crystallization and X-ray structure determination. Crystallization was per-} formed by sitting-drop vapour diffusion. Screening of $0.3 \mu \mathrm{L}$ of $1 \mathrm{mg} / \mathrm{mL} \mathrm{TaFdcV}$ in $25 \mathrm{mM}$ HEPES, $150 \mathrm{mM} \mathrm{KCl}, \mathrm{pH} 6.8$, and $0.3 \mu \mathrm{L}$ of reservoir solution at $4{ }^{\circ} \mathrm{C}$ resulted in a number of hits in the BCS plate from molecular dimensions. Seed stocks were used to reproduce $T a F d c$ wild-type crystals and co-crystals with 2-butynoic and crotonic acids in the BCS plate. Crystals were cryoprotected with PEG200 and flash-frozen in liquid nitrogen. AnFdc wild-type and variants were crystallized in $0.2 \mathrm{M}$ potassium thiocyanate, Bis-Tris propane $6.5,20 \% \mathrm{w} / \mathrm{v}$ PEG 3350 at $4^{\circ} \mathrm{C}^{16}$. Diffraction data were collected at Diamond beamlines and processed using the CCP4 ${ }^{21}$ suite version 7.1 (Supplementary Table 7). Phaser MR version 2.8.3 was used to perform molecular replacement using 4ZA4 [https:// doi.org/10.2210/pdb4ZA4/pdb] as a model. Refinement was carried out with REFMAC $^{22}$ and manual rebuilding in $\operatorname{COOT}^{23}$ version 0.9.5. Ligand definitions and coordinates were generated with AceDRG ${ }^{24}$.

In vitro isobutene assay comparing TaFdc and AnFdc variants. All variants were grown in BL21(DE3) cells with a pETDuet plasmid with the Fdc variant (N-terminal 6-His-tag) in the first multiple cloning site and UbiX (untagged, wild-type from E. coli K12) in second multiple cloning site. The cells were grown in a ZYM-5052 autoinducing medium $\left(30^{\circ} \mathrm{C}\right.$ for $6 \mathrm{~h}$, followed by $18^{\circ} \mathrm{C}$ for $\left.24 \mathrm{~h}\right)$. The Fdc variants were purified with Protino ${ }^{\circ} \mathrm{Ni}-\mathrm{IDA}$ column and stored at $-80^{\circ} \mathrm{C}$ (in $50 \mathrm{mM}$ Tris- $\mathrm{HCl} \mathrm{pH}$ $7.5,1 \mathrm{mM} \mathrm{MnCl}, 20 \mathrm{mM} \mathrm{NaCl}, 200 \mathrm{mM} \mathrm{KCl}, 10 \%$ glycerol). Decarboxylation of 3-methylcrotonate was set up in triplicates in $50 \mathrm{mM}$ Tris- $\mathrm{HCl} \mathrm{pH} \mathrm{7.5,} 1 \mathrm{mM} \mathrm{MnCl}_{2}$, $20 \mathrm{mM} \mathrm{NaCl}, 200 \mathrm{mM} \mathrm{KCl}$ with $10 \mathrm{mM} 3$-methylcrotonate and $0.3 \mathrm{mg} / \mathrm{mL}$ enzyme in DW384 plates ( $40 \mu \mathrm{L}$ per well, sealed with foil sheet). Isobutene production was measured from headspace by gas chromatography after 2 and $4 \mathrm{~h}$.

In vitro isobutene assay comparing TaFdc, ScMVD and PtM3K. An equal amount of E. coli cells containing either empty pETDuet (as control) or one of the following plasmids: pETDuet TaFdc_UbiX, pETDuet TaFdcV_UbiX, pETDuet
PtM3K (P. torridus mevalonate 3-kinase, Uniprot: Q6KZB1), pETDuet ScMVD ( $S$ cerevisiae MVD, Uniprot: P32377) or pETDuet PtM3K-ScMVD, were lysed in $50 \mathrm{mM}$ Tris- $\mathrm{HCl} \mathrm{pH}$ 7.5, $20 \mathrm{mM} \mathrm{KCl}, 2 \mathrm{mM} \mathrm{MgCl}_{2}, 1 \mathrm{~g} / \mathrm{L}$ lysozyme, $0.03 \mathrm{~g} / \mathrm{L}$ DNAse for $1 \mathrm{~h}$ at $37^{\circ} \mathrm{C}$. A total of $150 \mu \mathrm{L}$ of lysate was transferred to a $2 \mathrm{~mL} \mathrm{GC}$ vial and $\mathrm{MgCl}_{2}$ (10 mM final concentration) was added. Substrates were added to $50 \mathrm{mM}$ final concentration and $200 \mu \mathrm{L}$ total volume, and consisted of either 3-

hydroxyisovalerate/ATP, 3-phosphonooxy-isovalerate/ADP or 3-methylcrotonate. Following $4 \mathrm{~h}$ of incubation at either 37 or $50^{\circ} \mathrm{C}$, the reaction mixture was inactivated by incubation at $90^{\circ} \mathrm{C}$ for $5 \mathrm{~min}$. GC analysis of the gas phase was carried out as described above to determine isobutene levels produced. All reactions were carried out in duplicates.

DFT calculations. $\mathrm{TaFdcV}$ active site cluster model with crotonic (365 atoms) and 3 -methylcrotonic acid (368 atoms) was built based on the $\mathrm{TaFdcV}$ crystal structure with crotonic acid bound as Int3 adduct (Supplementary Fig. 9) and modelled at the B3LYP/6-31 G $(d, p)$ level of theory with the D3 version of Grimme's dispersion with Becke-Johnson damping and a generic polarizable continuum with $\varepsilon=5.7$ using the polarizable continuum model ${ }^{25} \cdot \mathrm{C}_{\alpha}-\mathrm{C}_{1}$, and $\mathrm{C}_{\beta}-\mathrm{C}_{4 \mathrm{a}}$ bonds were both fixed for any single DFT optimization and substrate release was modelled using Gaussian 09 revision D.01. by lengthening one bond by $0.05 \AA$ at a time, resulting in a 3D energy landscape consisting of $~ 900$ DFT optimized models (for 3-methylcrotonic acid).

Reporting summary. Further information on research design is available in the Nature Research Reporting Summary linked to this article.

\section{Data availability}

Data supporting the findings of this work are available within the paper and its Supplementary Information files. A reporting summary for this Article is available as a Supplementary Information file. Crystal structure data that support the findings of this 
study have been deposited in the PDB with the accession codes 7NEY, 7NF0, 7NF1, 7NF2, 7NF3 and 7NF4. Input and output files for DFT calculations are available at Zenodo [https://doi.org/10.5281/zenodo.5137885]. Source data are provided with this paper.

Received: 2 March 2021; Accepted: 29 July 2021;

Published online: 06 September 2021

References

1. Lee, S. Y. et al. A comprehensive metabolic map for production of bio-based chemicals. Nat. Catal. 2, 18-33 (2019).

2. Van Leeuwen, B. N. M., Van Der Wulp, A. M., Duijnstee, I., Van Maris, A. J. A. \& Straathof, A. J. J. Fermentative production of isobutene. Appl. Microbiol. Biotechnol. 93, 1377-1387 (2012).

3. Wilson, J., Gering, S., Pinard, J., Lucas, R. \& Briggs, B. R. Bio-production of gaseous alkenes: ethylene, isoprene, isobutene. Biotechnol. Biofuels 11, 1-11 (2018).

4. Fukuda, H., Fujii, T. \& Ogawa, T. Microbial production of c3- and c4hydrocarbons under aerobic conditions. Agric. Biol. Chem. 48, 1679-1682 (1984).

5. Fujii, T., Ogawa, T. \& Fukuda, H. Isobutene production by Rhodotorula minuta. Appl. Microbiol. Biotechnol. 25, 430-433 (1987).

6. Fukuda, H. et al. Reconstitution of the isobutene-forming reaction catalyzed by cytochrome P450 and P450 reductase from Rhodotorula minuta: Decarboxylation with the formation of isobutene. Biochem. Biophys. Res. Commun. 201, 516-522 (1994).

7. Gogerty, D. S. \& Bobik, T. A. Formation of isobutene from 3-hydroxy-3methylbutyrate by diphosphomevalonate decarboxylase. Appl. Environ. Microbiol. 76, 8004-8010 (2010).

8. Marliere, P. Production of alkenes by enzymatic decarboxylation of 3-hydroxyalkanoic acids. Patent WO2010001078 (2010) https://doi.org/ 10.1016/j.(73).

9. Rossoni, L., Hall, S. J., Eastham, G., Licence, P. \& Stephens, G. The putative mevalonate diphosphate decarboxylase from Picrophilus torridus is in reality a mevalonate-3-kinase with high potential for bioproduction of isobutene. Appl. Environ. Microbiol. 81, 2625-2634 (2015).

10. Marliere, P. Production of alkenes by combined enzymatic conversion of 3-hydroxyalkanoic acids. Patent WO2012052427 (2012).

11. Payne, K. A. P. et al. New cofactor supports $\alpha, \beta$-unsaturated acid decarboxylation via 1,3-dipolar cycloaddition. Nature 522, 497-501 (2015).

12. White, M. D. et al. UbiX is a flavin prenyltransferase required for bacterial ubiquinone biosynthesis. Nature 522, 502-506 (2015).

13. Marshall, S. A., Payne, K. A. P. \& Leys, D. The UbiX-UbiD system: the biosynthesis and use of prenylated flavin (prFMN). Arch. Biochem. Biophys. 632, 209-221 (2017).

14. Payne, K. A. P. et al. Enzymatic carboxylation of 2-furoic acid yields 2,5furandicarboxylic acid (FDCA). ACS Catal. 9, 2854-2865 (2019).

15. Ferguson, K. L., Eschweiler, J. D., Ruotolo, B. T. \& Marsh, E. N. G. Evidence for a 1,3-dipolar cyclo-addition mechanism in the decarboxylation of phenylacrylic acids catalyzed by ferulic acid decarboxylase. J. Am. Chem. Soc. 139, 10972-10975 (2017).

16. Bailey, S. S. et al. The role of conserved residues in Fdc decarboxylase in prenylated flavin mononucleotide oxidative maturation, cofactor isomerization, and catalysis. J. Biol. Chem. 293, 2272-2287 (2018).

17. Bailey, S. S. et al. Enzymatic control of cycloadduct conformation ensures reversible 1,3-dipolar cycloaddition in a prFMN-dependent decarboxylase. Nat. Chem. 11, 1049-1057 (2019).

18. Aleku, G. A. et al. Enzymatic C-H activation of aromatic compounds through CO2 fixation. Nat. Chem. Biol. 16, 1255-1260 (2020).

19. Hayakawa, H. et al. Modified mevalonate pathway of the archaeon Aeropyrum pernix proceeds via trans-anhydromevalonate 5-phosphate. Proc. Natl Acad. Sci. USA 115, 10034-10039 (2018).

20. Mori, Y., Noda, S., Shirai, T. \& Kondo, A. Direct 1,3-butadiene biosynthesis in Escherichia coli via a tailored ferulic acid decarboxylase mutant. Nat. Commun. 12, 1-12 (2021).
21. Winn, M. D. et al. Overview of the CCP4 suite and current developments. Acta Crystallogr. Sect. D 67, 235-242 (2011)

22. Murshudov, G. N. et al. REFMAC5 for the refinement of macromolecular crystal structures. Acta Crystallogr. Sect. D 67, 355-367 (2011)

23. Emsley, P., Lohkamp, B., Scott, W. G. \& Cowtan, K. Features and development of Coot. Acta Crystallogr. Sect. D 66, 486-501 (2010).

24. Long, F. et al. AceDRG: a stereochemical description generator for ligands. Acta Crystallogr. Sect. D 73, 112-122 (2017).

25. Grimme, S., Ehrlich, S. \& Goerigk, L. Effect of the damping function in dispersion corrected density functional theory. J. Comput. Chem. 32, 1456-1465 (2011)

\section{Acknowledgements}

This work was supported financially by grant ERC pre-FAB 695013 (D.L.). We acknowledge assistance via use of the Manchester Protein Structure Facility and the Diamond Light Source (proposal nos. MX12788 and MX17773), which contributed to the results presented here. We also acknowledge the assistance given by IT Services and the use of the Computational Shared Facility at The University of Manchester. D.L. is a Royal Society Wolfson Merit Award holder.

\section{Author contributions}

B.V., F.S., M.A. and M.C. performed initial in vivo screening for isobutene activity, directed evolution of $\mathrm{TaFdc}$ and isobutene assays with $\mathrm{AnFdc}$ and $\mathrm{TaFdc}$ variants, including expression and purification. A.S. performed point mutagenesis, enzyme expression, purification and characterization by UV-Vis absorption, inhibition studies and the assay with hexenoic acid. A.S. performed crystallization and determined crystal structures with assistance from D.L., R.S. assisted with mass spectrometry. A.S. and S.H. performed computational studies. A.S. and D.L. wrote the initial draft of the manuscript. All authors discussed the results and commented on the manuscript. D.L. and M.A. initiated and coordinated the project.

\section{Competing interests}

B.V., F.S., M.A. and M.C. are employees of Global Bioenergies. Other authors declare no competing interests.

\section{Additional information}

Supplementary information The online version contains supplementary material available at https://doi.org/10.1038/s41467-021-25598-0.

Correspondence and requests for materials should be addressed to B.V. or D.L.

Peer review information Nature Communications thanks Brandon Briggs, Hisashi Hemmi, and Andrea Mattevi for their contributions to the peer review of this work. Peer review reports are available.

Reprints and permission information is available at http://www.nature.com/reprints

Publisher's note Springer Nature remains neutral with regard to jurisdictional claims in published maps and institutional affiliations.

Open Access This article is licensed under a Creative Commons Attribution 4.0 International License, which permits use, sharing, adaptation, distribution and reproduction in any medium or format, as long as you give appropriate credit to the original author(s) and the source, provide a link to the Creative Commons license, and indicate if changes were made. The images or other third party material in this article are included in the article's Creative Commons license, unless indicated otherwise in a credit line to the material. If material is not included in the article's Creative Commons license and your intended use is not permitted by statutory regulation or exceeds the permitted use, you will need to obtain permission directly from the copyright holder. To view a copy of this license, visit http://creativecommons.org/ licenses/by/4.0/.

(C) The Author(s) 2021 